Archive for

Organic Chemistry

Arkivoc 2018, part iii, 112-119

\title{
An alternative stereoselective synthesis of (-)-1-tetrahydropyrenophorol
}

\author{
Mahesh Madala, ${ }^{a, b}$ Balamurali Raman, ${ }^{a}$ K. V. Sastry, ${ }^{c}$ Sridhar Musulla, ${ }^{a, b}$ and Gattu Sridhar ${ }^{d}$ \\ ${ }^{a}$ GVK Biosciences Private Limited, Nacharam, IDA Mallapur, Hyderabad, Telangana 500 076, India \\ ${ }^{b}$ Department of Chemistry, JNT University, Hyderabad, Telangana 500 082, India \\ ${ }^{c}$ Teegala Krishna Reddy College of Pharmacy, Medbowli, Meerpet, Saroornagar, Hyderabad, \\ Telangana 500 097, India \\ ${ }^{d}$ Organic and Biomolecular Chemistry Division, CSIR-Indian Institute of Chemical Technology, \\ Hyderabad, Telangana 500 107, India \\ E-mail: mm1org@hotmail.com
}

Received 08-25-2017

Accepted 12-21-2017

Published on line $01-23-2018$

\section{Abstract}

Macrodiolides have become highly attractive target molecules because of their interesting structural features and biological properties, including antibacterial, antifungal, cytotoxic, and phytotoxic activity. A simple and efficient synthesis of the macrocyclic dilactone, (-)-1-tetrahydropyrenophorol, has been accomplished from commercially available compounds. The synthesis utilizes regioselective ring opening of a chiral epoxide, followed by asymmetric dihydroxylation and a Mitsunobu reaction for the construction of the macrolactone.

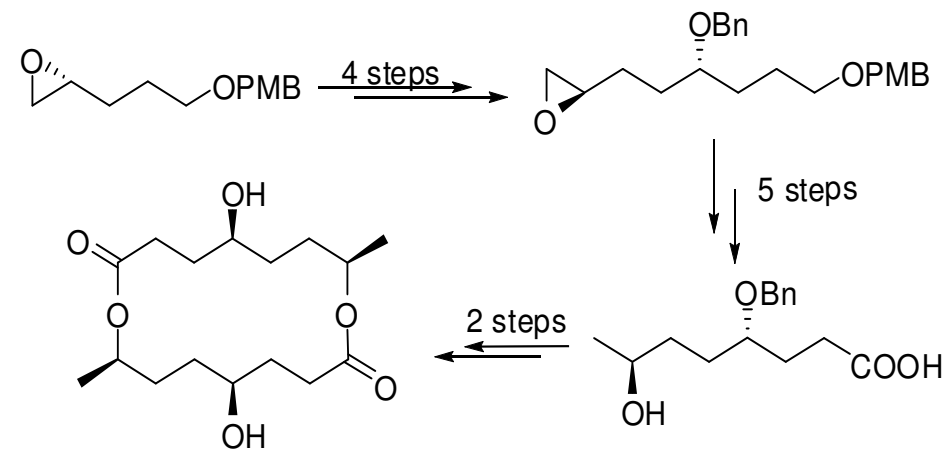

(-)-1-Tetrahydropyrenophorol

Keywords: (-)-1-Tetrahydropyrenophorol, macrodiolide, asymmetric dihydroxylation, cyclodimerisation, Mitsunobu reaction 


\section{Introduction}

Macrodiolides have become highly attractive target molecules to synthetic chemists in recent years because of their biological properties and interesting structural features. In nature, macrodiolides are found as both homodimers ${ }^{1-4}$, consisting of two identical units and showing $C_{2}$ symmetry, and heterodimers ${ }^{5}$, consisting of two different units. Many of these macrodiolides (both homo and hetero) exhibit potent biological activities, such as antibacterial, antifungal, cytotoxic, and phytotoxic activity. ${ }^{6,9}$

(-)-1-Tetrahydropyrenophorol (Fig. 1 ) is an example of a $\mathrm{C}_{2}$-symmetric macrodiolide. It was isolated from the ethyl-acetate extract of a culture of an endophytic Phoma sp. isolated from the plant Fagonia cretica. It exhibits good herbicidal and algicidal and moderate fungicidal activities. The relative configuration of (-)-1tetrahydropyrenophorol (1) was confirmed by X-ray single-crystal analysis. Its absolute configuration was determined by solid-state time-dependent density-functional theory (TDDFT) CD methodology. ${ }^{2}$ Recently, a synthesis of (-)-1-tetrahydropyrenophorol was reported by Pratapareddy et al., ${ }^{10}$ while Trost and Quintard ${ }^{11}$ reported the total synthesis of (+)-tetrahydropyrenophorol.

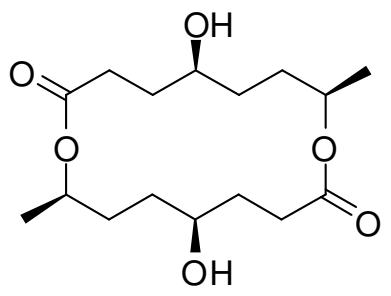

(-)-1-Tetrahydropyrenophorol

Figure 1

\section{Results and Discussion}

In continuation of our work on the synthesis of biologically-active natural products, ${ }^{12}$ we report herein an efficient straightforward and concise total synthesis of (-)-1-tetrahydropyrenophorol starting from commercially available starting materials.

As depicted in Scheme 1, retrosynthetic analysis of (1) envisioned that it could be obtained from the hydroxy-acid (2) via cyclodimerisation under Mitsunobu reaction conditions, followed by deprotection of the benzyl ether. The hydroxy-acid (2) could easily be prepared from the diol (3), which in turn could be prepared from the known chiral epoxide (4), all by simple chemical transformations.

Synthesis of (-)-1-tetrahydropyrenophorol (1) (Scheme 2) began with the reported chiral $p$ methoxybenzyloxy-epoxide (4). ${ }^{13}$ Regioselective ring-opening of (4) by allyl magnesium chloride in the presence of Cul yielded the alcohol (5) in $87 \%$ yield, which, on subsequent benzylation with $\mathrm{NaH}$ and benzyl bromide at $0{ }^{\circ} \mathrm{C}$, gave (6) in $91 \%$ yield. The terminal olefin group in (6) was subjected to asymmetric dihydroxylation with $A D-m i x-\beta$ in $t-\mathrm{BuOH} / \mathrm{H}_{2} \mathrm{O}$ to afford diol (3) in $79 \%$ yield (d.r. 9:1). ${ }^{14}$ Selective monotosylation of the diol (3) using $\mathrm{TsCl}$ and $\mathrm{Et}_{3} \mathrm{~N}$ in $\mathrm{CH}_{2} \mathrm{Cl}_{2}$, followed by cyclization of the resulting monotosylate (3a) in the presence of $\mathrm{K}_{2} \mathrm{CO}_{3}$ in $\mathrm{MeOH}$, afforded the chiral epoxide (7) in $77 \%$ yield. 


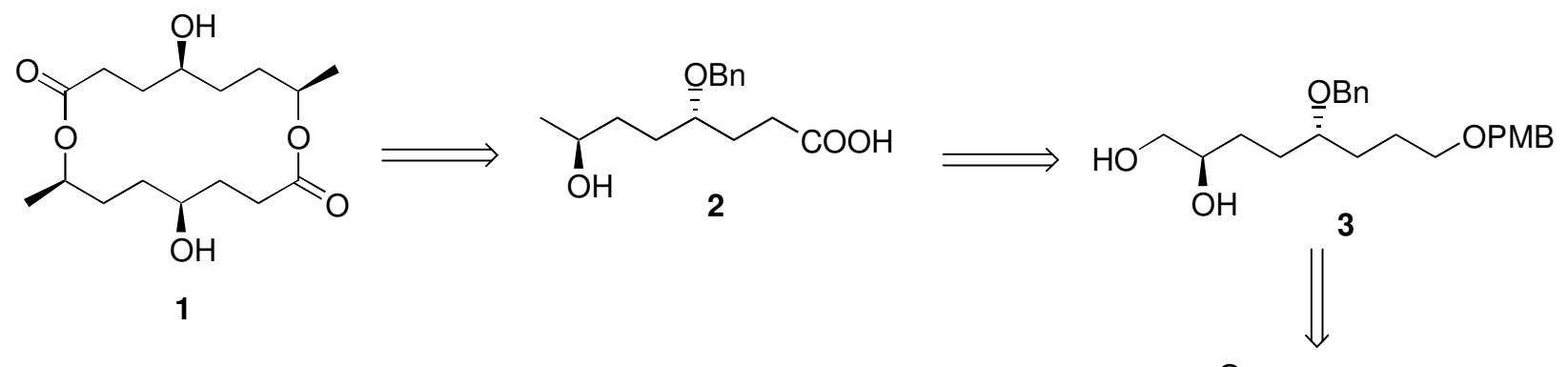<smiles>[134S]OCCCC1CO1</smiles>

4

Scheme 1. Retrosynthesis model for (-)-1-tetrahydropyrenophorol (1) from p-methoxybenzyloxy-epoxide (4).
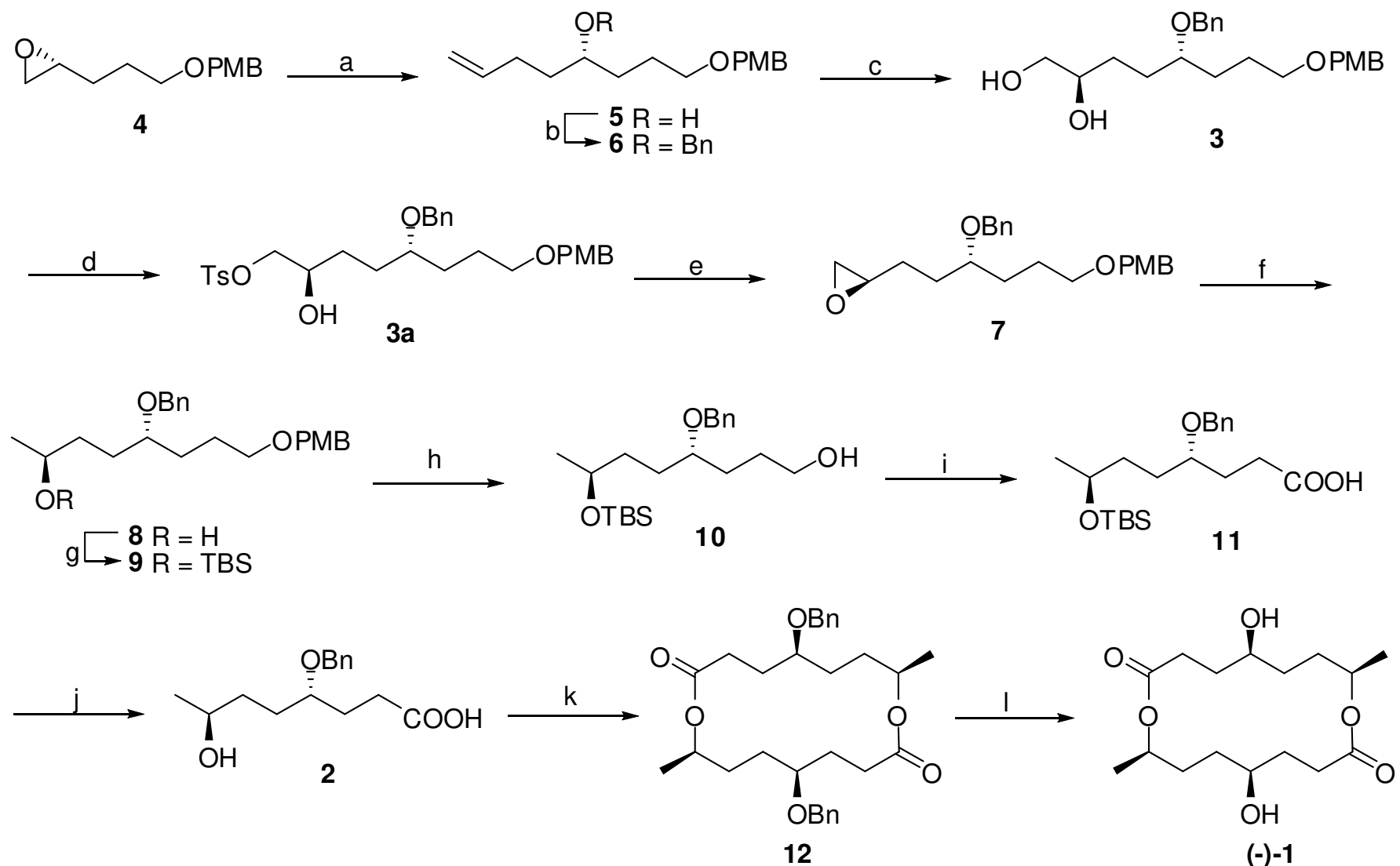

Scheme 2. Preparative route to (-)-1-tetrahydropyrenophorol (1). Reagents and conditions: (a) allyl chloride,

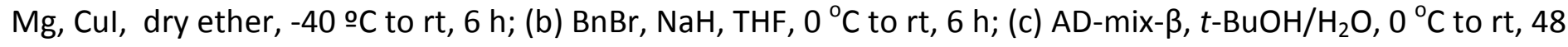
$\mathrm{h}$; (d) $p$ - TsCl, $\mathrm{Et}_{3} \mathrm{~N}$, rt, $2 \mathrm{~h}$; (e) $\mathrm{K}_{2} \mathrm{CO}_{3}, \mathrm{MeOH}, \mathrm{rt}, 1 \mathrm{~h}$; (f) LAH, THF, $0{ }^{\circ} \mathrm{C}$ to rt, $3 \mathrm{~h}$; (g) TBSCl, imidazole, $\mathrm{CH}_{2} \mathrm{Cl}_{2}, \mathrm{rt}, 4$ h; (h) DDQ, $\mathrm{CH}_{2} \mathrm{Cl}_{2}: \mathrm{H}_{2} \mathrm{O}$ (19:1), rt, 3 h; (i) TEMPO, [bis(acetoxy)iodo]benzene, aq. $\mathrm{CH}_{2} \mathrm{Cl}_{2}, 0$ 으, 1 h; (j) TBAF, THF, $0{ }^{\circ} \mathrm{C}$ to rt, $3 \mathrm{~h}$; (k) Ph $3{ }_{3} \mathrm{P}, \mathrm{DEAD}$, toluene:THF (10:1) $-20{ }^{\circ} \mathrm{C}, 10 \mathrm{~h}$; (I) $\mathrm{TiCl}_{4}, \mathrm{CH}_{2} \mathrm{Cl}_{2}, 0{ }^{\circ} \mathrm{C}$ to rt, $1 \mathrm{~h}$.

Regioselective opening of the epoxide (7) with LAH in dry THF furnished the alcohol (8) in $87 \%$ yield, which, on subsequent masking with t-butyldimethylsilyl chloride (TBSCl) in the presence of imidazole at $0{ }^{\circ} \mathrm{C}$, afforded (9) in $91 \%$ yield. Next, selective cleavage of the $p$-methoxybenzyloxy (PMB) ether from compound (9), in the presence of 2,3-dichloro-5,6-dicyano-1,4-benzoquinone (DDQ) in aq. $\mathrm{CH}_{2} \mathrm{Cl}_{2}$, gave alcohol (10) in $86 \%$ 
yield. The alcohol (10) was then oxidized following treatment with 2,2,6,6-tetramethyl-1-piperidinyloxy (TEMPO) and [bis(acetoxy)iodo]benzene in aq. $\mathrm{CH}_{2} \mathrm{Cl}_{2}$, affording the corresponding carboxylic acid (11) in $75 \%$ yield, which, on desilylation with tetra-n-butylammonium fluoride (TBAF) in dry THF, gave the hydroxy-acid (2) in $86 \%$ yield.

Following the successful synthesis of the hydroxyacid (2), it was subjected to cyclodimerisation under Mitsunobu reaction conditions $\left[\mathrm{Ph}_{3} \mathrm{P} \text { and diethyl azodicarboxylate (DEAD) }\right]^{15}$ at $-20{ }^{\circ} \mathrm{C}$ for $10 \mathrm{~h}$ to furnish (12) in $59 \%$ yield. Finally, debenzylation of (12) with $\mathrm{TiCl}_{4}$ in $\mathrm{CH}_{2} \mathrm{Cl}_{2}$ for $3 \mathrm{~h}$ afforded (-)-1-tetrahydropyrenophorol (1) in $77 \%$ yield. The spectroscopic data $\left({ }^{1} \mathrm{H}\right.$ and $\left.{ }^{13} \mathrm{C} N \mathrm{NMR}\right)$ and specific optical rotation $\left([\alpha]_{\mathrm{D}}^{25}-70.3\right.$ (c 0.54 , $\left.\mathrm{CHCl}_{3}\right)$ ) of (1) were in good agreement with the reported values $\left([\alpha]_{\mathrm{D}}^{25}-68\left(c 0.14, \mathrm{CHCl}_{3}\right)\right) .{ }^{10}$

\section{Conclusions}

A concise stereoselective total synthesis of the macrodiolide, (-)-1-tetrahydropyrenophorol, was accomplished using an efficient combination of regioselective opening of a known chiral epoxide, subsequent asymmetric dihydroxylation, and Mitsunobu reaction.

\section{Experimental Section}

General. Solvents were dried over standard drying agents or freshly distilled prior to use. Chemicals were purchased and used without further purification. All column chromatographic separations were performed using silica gel (60-120 mesh). Organic solutions were dried over anhydrous $\mathrm{Na}_{2} \mathrm{SO}_{4}$ and concentrated below $40{ }^{\circ} \mathrm{C}$ in vacuo. ${ }^{1} \mathrm{H}$ NMR spectra were acquired at $300 \mathrm{MHz}, 500 \mathrm{MHz}$ and $600 \mathrm{MHz}$ while ${ }^{13} \mathrm{C}$ NMR spectra were acquired at $75 \mathrm{MHz}$ and $125 \mathrm{MHz}$, both with TMS as internal standard for solutions in $\mathrm{CDCl}_{3}$. J values are given in $\mathrm{Hz}$. The following abbreviations are used in reporting NMR data: $s$, singlet; brs, broad singlet; d, doublet; dd, doublet of doublets; m, multiplet; and t, triplet. IR spectra were recorded on an FT IR spectrophotometer with $\mathrm{NaCl}$ optics. Optical rotations were measured on a digital polarimeter at $25^{\circ} \mathrm{C}$. Mass spectra were recorded with a direct inlet system or LC by MSD trap SL. The HRMS data were obtained using Q-TOF mass spectrometry.

(S)-1-(4-Methoxybenzyloxy)oct-7-en-4-ol (5). To a stirred solution of epoxide (4) (4.6 g, $20.72 \mathrm{mmol})$ in dry diethyl ether $(100 \mathrm{~mL})$, copper $(\mathrm{l})$ iodide $(1.96 \mathrm{~g}, 10.35 \mathrm{mmol})$ was added and the mixture was cooled to $-40{ }^{\circ} \mathrm{C}$. A solution of allylmagnesium chloride in ether [generated from $\mathrm{Mg}(1.49 \mathrm{~g}, 62.16 \mathrm{mmol})$ and allyl chloride $(2.13 \mathrm{~mL}, 24.86 \mathrm{mmol}$ in $50 \mathrm{~mL}$ ether)] was added. After the addition was complete, the mixture was stirred for $6 \mathrm{~h}$ and then quenched with aq. $\mathrm{NH}_{4} \mathrm{Cl}$ solution $(30 \mathrm{~mL})$ dropwise. The residue was filtered using through celite and the filtrate was extracted with EtOAc $(2 \times 30 \mathrm{~mL})$. The combined organic layers were dried $\left(\mathrm{Na}_{2} \mathrm{SO}_{4}\right)$ and evaporated under reduced pressure. The crude product was purified by column chromatography (silica gel, 60-120 mesh, $12 \%$ EtOAc in pet. ether) to furnish (5) $(4.75 \mathrm{~g}, 87 \%)$ as a yellow liquid. $[\alpha]_{\mathrm{D}}^{25}+11.3$ (c 1.5, $\mathrm{CHCl}_{3}$ ); IR (neat): 3457, 3077, 2988, 2929, 1622, 1375, 1213, $854 \mathrm{~cm}^{-1} ;{ }^{1} \mathrm{H}$ NMR (CDCl, $\left.300 \mathrm{MHz}\right): \delta 7.19$ (d, $2 \mathrm{H}, J 8.0 \mathrm{~Hz}), 6.89(\mathrm{~d}, 2 \mathrm{H}, J 8.0 \mathrm{~Hz}), 5.83(\mathrm{~m}, 1 \mathrm{H}), 4.99(\mathrm{~m}, 2 \mathrm{H}), 4.47(\mathrm{~s}, 2 \mathrm{H}), 3.79(\mathrm{~s}, 3 \mathrm{H}), 3.68-3.57(\mathrm{~m}, 1 \mathrm{H}), 3.49$ $(\mathrm{t}, 2 \mathrm{H}, J 8.0 \mathrm{~Hz}), 2.81$ (brs, $1 \mathrm{H},-\mathrm{OH}), 2.16-2.08(\mathrm{~m}, 2 \mathrm{H}), 1.71-1.59(\mathrm{~m}, 2 \mathrm{H}), 1.41-1.30(\mathrm{~m}, 4 \mathrm{H}) ;{ }^{13} \mathrm{C} N M R\left(\mathrm{CDCl}_{3}\right.$, 
$75 \mathrm{MHz}$ : $\delta$ 159.3, 134.5, 130.3, 129.6, 114.6, 113.3, 76.1, 69.3, 68.3, 56.2, 37.2, 35.2, 31.2, 30.9; ESIMS: 287 $(\mathrm{M}+\mathrm{Na})^{+} \mathrm{HRMS}(\mathrm{ESI}): \mathrm{m} / \mathrm{z}$ calcd for $\mathrm{C}_{16} \mathrm{H}_{24} \mathrm{O}_{3} \mathrm{Na}$ : 287.1626; found: $287.1631[\mathrm{M}+\mathrm{Na}]^{+}$.

(S)-1-((4-(Benzyloxy)oct-7-enyloxy)methyl)-4-methoxybenzene (6). To a cooled $\left(0{ }^{\circ} \mathrm{C}\right)$ solution of (5) (4.4 g, $16.66 \mathrm{mmol})$ in dry THF $(15 \mathrm{~mL}), \mathrm{NaH}(1.2 \mathrm{~g}, 49.98 \mathrm{mmol})$ was added, stirred for $30 \mathrm{~min}$ and treated with a solution of benzyl bromide $(2.36 \mathrm{~mL}, 19.92 \mathrm{mmol})$ in dry THF $(10 \mathrm{~mL})$. After stirring at room temperature for 6 $h$, the reaction mixture was quenched with sat. $\mathrm{NH}_{4} \mathrm{Cl}$ solution $(15 \mathrm{~mL})$ and extracted with ethyl acetate $(2 \times 50$ $\mathrm{mL})$. The organic layers were washed with water $(2 \times 30 \mathrm{~mL})$, brine $(30 \mathrm{~mL})$ and dried $\left(\mathrm{Na}_{2} \mathrm{SO}_{4}\right)$. The solvent was evaporated under reduced pressure and the residue purified by column chromatography (60-120 silica gel, $8 \%$ EtOAc in pet. ether) to furnish (6) $\left(5.25 \mathrm{~g}, 91 \%\right.$ ) as a yellow liquid. $[\alpha]_{\mathrm{D}}^{25}+141.7$ (c 1.2, $\mathrm{CHCl}_{3}$ ); IR (neat): 3071, 2989, 2935, 1617, 1515, 1248, $1061 \mathrm{~cm}^{-1}{ }^{1} \mathrm{H}$ NMR $\left(\mathrm{CDCl}_{3}, 300 \mathrm{MHz}\right): \delta$ 7.41-7.29 (m, 5H), 7.19 (d, $2 \mathrm{H}, J$ $8.5 \mathrm{~Hz}), 6.77(\mathrm{~d}, 2 \mathrm{H}, \mathrm{J} 8.4 \mathrm{~Hz}), 5.79(\mathrm{~m}, 1 \mathrm{H}), 5.01(\mathrm{~m}, 2 \mathrm{H}), 4.59(\mathrm{~d}, 1 \mathrm{H}, J 10.6 \mathrm{~Hz}), 4.49(\mathrm{~s}, 2 \mathrm{H}), 4.39(\mathrm{~d}, 1 \mathrm{H}, J 10.6$ $\mathrm{Hz}), 3.76(\mathrm{~s}, 3 \mathrm{H}), 3.54(\mathrm{t}, 2 \mathrm{H}, \mathrm{J} 7.1 \mathrm{~Hz}), 3.46-3.32(\mathrm{~m}, 1 \mathrm{H}), 2.21-2.11(\mathrm{~m}, 2 \mathrm{H}), 1.63-1.31(\mathrm{~m}, 6 \mathrm{H}) ;{ }^{13} \mathrm{C} \mathrm{NMR} \mathrm{CDCl}_{3}$, $75 \mathrm{MHz}$ ): $\delta$ 159.1, 138.3, 136.6, 129.8, 129.0, 128.4, 128.1, 127.7, 114.7, 113.1, 79.1, 76.0, 73.1, 72.2, 56.1, 33.4, 32.4, 31.8, 29.8; HRMS (ESI): $m / z$ calcd for $\mathrm{C}_{23} \mathrm{H}_{30} \mathrm{O}_{3} \mathrm{Na}$ : 377.2091; found: 377.2096 [M+Na] ${ }^{+}$.

(2R,5R)-5-(Benzyloxy)-8-(4-methoxybenzyloxy)octane-1,2-diol (3). A mixture of ADmix- $\beta$ (11.20 g, 14.40 $\mathrm{mmol})$ in $50 \mathrm{~mL}$ of $t-\mathrm{BuOH} / \mathrm{H}_{2} \mathrm{O}(1: 1 \mathrm{v}: \mathrm{v})$ was stirred at $\mathrm{rt}$ for $15 \mathrm{~min}$, and then cooled to $0{ }^{\circ} \mathrm{C}$. To this solution was added olefin (6) $(5.1 \mathrm{~g}, 14.40 \mathrm{mmol})$. The reaction mixture was stirred at $0{ }^{\circ} \mathrm{C}$ for $48 \mathrm{~h}$ and then quenched with $\mathrm{Na}_{2} \mathrm{SO}_{3}(7.5 \mathrm{~g})$ at $0{ }^{\circ} \mathrm{C}$ within $0.5 \mathrm{~h}$. EtOAc $(50 \mathrm{~mL})$ was added to the reaction mixture, and the aqueous layer was further extracted with EtOAc $(2 \times 50 \mathrm{~mL})$. The combined organic layers were dried over $\mathrm{Na}_{2} \mathrm{SO}_{4} \mathrm{and}$ the solvents were evaporated. The crude product was purified by column chromatography on silica gel $(30 \%$ EtOAc in pet. ether) to give the corresponding diol (3) $(4.41 \mathrm{~g}, 79 \%)$ as a colorless oil: $[\alpha]_{\mathrm{D}}^{25}-66.8\left(\mathrm{c} 0.5, \mathrm{CHCl}_{3}\right)$; IR (neat): 3457, 3069, 2952, 2846, 1613, 1518, 1247, 1079, 936, $707 \mathrm{~cm}^{-1}{ }^{1} \mathrm{H} \mathrm{NMR}\left(\mathrm{CDCl}_{3}, 300 \mathrm{MHz}\right): \delta 7.32$ (m, $5 \mathrm{H}), 7.21(\mathrm{~d}, 2 \mathrm{H}, J 8.3 \mathrm{~Hz}), 6.81(\mathrm{~d}, 2 \mathrm{H}, J 8.4 \mathrm{~Hz}), 4.60(\mathrm{~d}, 1 \mathrm{H}, J 11.0 \mathrm{~Hz}), 4.48(\mathrm{~d}, 1 \mathrm{H}, \mathrm{J} 11.0 \mathrm{~Hz}), 4.38(\mathrm{~s}, 2 \mathrm{H}), 3.78$ $(\mathrm{s}, 3 \mathrm{H}), 3.69-3.61(\mathrm{~m}, 3 \mathrm{H}), 3.40-3.26(\mathrm{~m}, 3 \mathrm{H}), 3.01$ (brs, 1H, $-\mathrm{OH}), 2.42$ (brs, $1 \mathrm{H},-\mathrm{OH}), 1.63-1.57(\mathrm{~m}, 2 \mathrm{H}), 1.49-$ $1.31(\mathrm{~m}, 5 \mathrm{H}), 1.23-1.10(\mathrm{~m}, 1 \mathrm{H}) ;{ }^{13} \mathrm{C} \mathrm{NMR}\left(\mathrm{CDCl}_{3}, 75 \mathrm{MHz}\right): \delta 158.7,136.6,130.1,129.8,129.5,129.3,128.8$, $113.9,79.1,76.2,73.3,72.4,68.3,56.7,32.2$, 31.6, 31.0, 29.8; HRMS (ESI): $m / z$ calcd for $\mathrm{C}_{23} \mathrm{H}_{32} \mathrm{O}_{5} \mathrm{Na}: 411.2148$; found: $411.2141[\mathrm{M}+\mathrm{Na}]^{+}$.

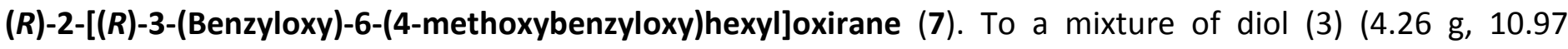
$\mathrm{mmol}$ ) in dry dichloromethane $(30 \mathrm{~mL})$ was added $p$-toluenesulfonyl chloride (2.08 g, $10.97 \mathrm{mmol})$, triethylamine $(2.2 \mathrm{~mL}, 16.45 \mathrm{mmol})$. The reaction was stirred for $2 \mathrm{~h}$ at room temperature under nitrogen and was monitored by TLC. After completion of the reaction, the mixture was quenched by adding water. The solution was extracted with $\mathrm{CH}_{2} \mathrm{Cl}_{2}(3 \times 20 \mathrm{~mL})$ and the combined organic phase washed with water, dried $\left(\mathrm{Na}_{2} \mathrm{SO}_{4}\right)$, and concentrated to give (3a) as a yellow liquid which was immediately used for the next step without any purification.

To the above crude mixture in $\mathrm{MeOH}$ at $0{ }^{\circ} \mathrm{C}$ was added $\mathrm{K}_{2} \mathrm{CO}_{3}(2.2 \mathrm{~g}, 16.45 \mathrm{mmol})$. The resultant mixture was stirred for $1 \mathrm{~h}$ at the same temperature. After completion of the reaction (as indicated by TLC), the reaction was quenched by the addition of pieces of ice, and the methanol was evaporated off. The concentrated reaction mixture was then extracted with ethyl acetate $(3 \times 20 \mathrm{~mL})$, the combined organic layers were washed with brine, dried $\left(\mathrm{Na}_{2} \mathrm{SO}_{4}\right)$, and concentrated. Column chromatography of the crude product using $10 \%$ EtOAc in pet. ether gave the epoxide (7) $(3.12 \mathrm{~g}, 77 \%)$ as a colorless liquid. $[\alpha]_{\mathrm{D}}^{25}-74.8\left(c 0.9, \mathrm{CHCl}_{3}\right) ;{ }^{1} \mathrm{HNMR}(300 \mathrm{MHz}$, $\left.\mathrm{CDCl}_{3}\right): \delta 7.33-7.23(\mathrm{~m}, 5 \mathrm{H}), 7.14(\mathrm{~d}, 2 \mathrm{H}, J 8.6 \mathrm{~Hz}), 6.80(\mathrm{~d}, 2 \mathrm{H}, J 8.6 \mathrm{~Hz}), 4.52(\mathrm{~d}, 1 \mathrm{H}, J 10.9 \mathrm{~Hz}), 4.41(\mathrm{~s}, 2 \mathrm{H}), 4.32$ (d, $1 \mathrm{H}, J 10.9 \mathrm{~Hz}), 3.68(\mathrm{~s}, 3 \mathrm{H}), 3.51(\mathrm{t}, 2 \mathrm{H}, J 6.8 \mathrm{~Hz}), 3.42-3.31(\mathrm{~m}, 1 \mathrm{H}), 2.91-2.86(\mathrm{~m}, 1 \mathrm{H}), 2.67(\mathrm{dd}, 1 \mathrm{H}, J 5.1,3.2$ $\mathrm{Hz}), 2.44(\mathrm{dd}, 1 \mathrm{H}, \mathrm{J} 5.1,3.0 \mathrm{~Hz}), 1.69-1.58(\mathrm{~m}, 2 \mathrm{H}), 1.41-1.21(\mathrm{~m}, 6 \mathrm{H}) ;{ }^{13} \mathrm{C} \mathrm{NMR}\left(\mathrm{CDCl}_{3}, 75 \mathrm{MHz}\right): \delta 159.4,138.2$, 
130.1, 128.6, 128.4, 128.0, 127.8, 114.1, 78.9, 76.6, 73.4, 72.0, 56.1, 54.7, 45.1, 31.3, 30.8, 30.2, 29.8; ESIMS: $393(\mathrm{M}+\mathrm{Na})^{+}$. HRMS (ESI): $\mathrm{m} / z$ calcd for $\mathrm{C}_{23} \mathrm{H}_{30} \mathrm{O}_{4} \mathrm{Na}$ : 393.2042; found: $393.2047[\mathrm{M}+\mathrm{Na}]^{+}$.

(2S,5R)-5-(Benzyloxy)-8-(4-methoxybenzyloxy)octan-2-ol (8). To a stirred suspension of LAH (0.46 g, 12.16 $\mathrm{mmol})$ in dry THF $(5 \mathrm{~mL})$, a solution of (7) $(3.0 \mathrm{~g}, 8.10 \mathrm{mmol})$ in dry THF $(10 \mathrm{~mL})$ was added dropwise at $0{ }^{\circ} \mathrm{C}$ under nitrogen atmosphere. The resulting mixture was stirred for $3 \mathrm{~h}$ at room temperature. The reaction mixture was cooled to $0{ }^{\circ} \mathrm{C}$, treated with saturated aq. $\mathrm{Na}_{2} \mathrm{SO}_{4}$ solution, filtered, and the filtrate was dried $\left(\mathrm{Na}_{2} \mathrm{SO}_{4}\right)$ and concentrated. The residue was purified by column chromatography (60-120 Silica gel, $18 \%$ EtOAc in pet. ether) to give (8) $(2.62 \mathrm{~g}, 87 \%)$ as a colorless syrup. $[\alpha]_{\mathrm{D}}^{25}+28.1\left(c 0.49, \mathrm{CHCl}_{3}\right)$; IR (neat): 3448,2932 , $1611,1513,1455,1374,1093,928 \mathrm{~cm}^{-1} ;{ }^{1} \mathrm{H}$ NMR $\left(300 \mathrm{MHz}, \mathrm{CDCl}_{3}\right): \delta 7.37-7.26(\mathrm{~m}, 5 \mathrm{H}), 7.19(\mathrm{~d}, 2 \mathrm{H}, \mathrm{J} 8.7 \mathrm{~Hz})$, $6.82(\mathrm{~d}, 2 \mathrm{H}, \mathrm{J} 8.7 \mathrm{~Hz}), 4.56(\mathrm{~d}, 1 \mathrm{H}, \mathrm{J} 11.1 \mathrm{~Hz}), 4.48(\mathrm{~d}, 1 \mathrm{H}, \mathrm{J} 11.1 \mathrm{~Hz}), 4.41(\mathrm{~s}, 2 \mathrm{H}), 3.80-3.69(\mathrm{~m}, 1 \mathrm{H}), 3.67(\mathrm{~s}, 3 \mathrm{H})$, $3.55(\mathrm{t}, 2 \mathrm{H}, \mathrm{J}, 6.6 \mathrm{~Hz}), 3.48-3.32(\mathrm{~m}, 1 \mathrm{H}), 2.41$ (brs, $1 \mathrm{H},-\mathrm{OH}), 1.69-1.58(\mathrm{~m}, 2 \mathrm{H}), 1.47-1.33(\mathrm{~m}, 3 \mathrm{H}), 1.28-1.13(\mathrm{~m}$, 3H), 1.06 (d, 3H, J $6.3 \mathrm{~Hz}) ;{ }^{13} \mathrm{C} \mathrm{NMR}\left(\mathrm{CDCl}_{3}, 75 \mathrm{MHz}\right): \delta$ 159.7, 138.2, 129.8, 129.5, 129.1, 128.7, 128.4, 113.9, 79.2, 75.9, 73.2, 72.7, 69.3, 54.9, 37.3, 32.1, 31.3, 30.7, 25.4; ESIMS: $373(\mathrm{M}+\mathrm{H})^{+}$. HRMS (ESI): $\mathrm{m} / \mathrm{z}$ calcd for $\mathrm{C}_{23} \mathrm{H}_{32} \mathrm{O}_{4} \mathrm{Na}$ : 395.2195; found: $395.2211[\mathrm{M}+\mathrm{Na}]^{+}$.

[(2S,5R)-5-(Benzyloxy)-8-(4-methoxybenzyloxy)octan-2-yloxy](tert-butyl)dimethylsilane (9). A mixture of the alcohol (8) $(2.5 \mathrm{~g}, 6.72 \mathrm{mmol})$ and imidazole $(1.37 \mathrm{~g}, 20.16 \mathrm{mmol})$ in dry $\mathrm{CH}_{2} \mathrm{Cl}_{2}(20 \mathrm{~mL})$ was treated with $\mathrm{TBSCl}$ $(1.20 \mathrm{~g}, 8.06 \mathrm{mmol})$ at $0{ }^{\circ} \mathrm{C}$ under nitrogen atmosphere and stirred at room temperature for $4 \mathrm{~h}$. The reaction mixture was quenched with aq. $\mathrm{NH}_{4} \mathrm{Cl}$ solution $(20 \mathrm{~mL})$ and extracted with $\mathrm{CH}_{2} \mathrm{Cl}_{2}(2 \times 50 \mathrm{~mL})$. The combined extracts were washed with water $(30 \mathrm{~mL})$, brine $(30 \mathrm{~mL})$, dried $\left(\mathrm{Na}_{2} \mathrm{SO}_{4}\right)$ and concentrated. The residue was purified by column chromatography (60-120 silica gel, 12\% EtOAc in pet. ether) to furnish (9) (2.97 g, $91 \%)$ as a colorless liquid, $[\alpha]_{\mathrm{D}}^{25}-57.4$ (c 0.76, $\mathrm{CHCl}_{3}$ ); IR (neat): 3069, 2931, 2858, 1613, 1512, 1247, 1105, $1083,701 \mathrm{~cm}^{-1}$; ${ }^{1} \mathrm{H}$ NMR $\left(300 \mathrm{MHz}, \mathrm{CDCl}_{3}\right): \delta 7.34-7.22(\mathrm{~m}, 5 \mathrm{H}), 7.19(\mathrm{~d}, 2 \mathrm{H}, J 8.4 \mathrm{~Hz}), 6.79(\mathrm{~d}, 2 \mathrm{H}, J 8.4 \mathrm{~Hz}), 4.53(\mathrm{~d}, 1 \mathrm{H}, J 10.6$ $\mathrm{Hz})$, 4.43-4.27 (m, $3 \mathrm{H}), 3.68(\mathrm{~s}, 3 \mathrm{H}), 3.59-3.42(\mathrm{~m}, 1 \mathrm{H}), 3.40-3.27(\mathrm{~m}, 3 \mathrm{H}), 1.70-1.50(\mathrm{~m}, 4 \mathrm{H}), 1.49-1.31(\mathrm{~m}, 3 \mathrm{H})$, $1.22(\mathrm{~d}, 3 \mathrm{H}, \mathrm{J} 6.6 \mathrm{~Hz}), 1.18-1.05(\mathrm{~m}, 1 \mathrm{H}), 0.81(\mathrm{~s}, 9 \mathrm{H}), 0.16(\mathrm{~s}, 6 \mathrm{H}) .{ }^{13} \mathrm{C} \mathrm{NMR}\left(\mathrm{CDCl}_{3}, 75 \mathrm{MHz}\right): \delta 158.9,137.2$, 129.8, 128.8, 128.3, 127.9, 127.7, 113.8, 78.7, 75.9, 73.2, 71.6, 67.1, 55.9, 36.2, 33.3, 32.8, 31.6, 26.3, 24.1, 17.3, -4.1; ESIMS: $487(\mathrm{M}+\mathrm{H})^{+}$. HRMS (ESI): $\mathrm{m} / z$ calcd for $\mathrm{C}_{29} \mathrm{H}_{46} \mathrm{O}_{4} \mathrm{SiNa}: 509.3064$; found: 509.3055 [M+Na]+.

(4R,7S)-4-(Benzyloxy)-7-(tert-butyldimethylsilyloxy)octan-1-ol (10). To a solution of the silane (9) (2.76 g, 5.67 $\mathrm{mmol})$ in aq. $\mathrm{CH}_{2} \mathrm{Cl}_{2}(20 \mathrm{~mL}, 19: 1)$, DDQ $(1.54 \mathrm{~g}, 6.81 \mathrm{mmol})$ was added and stirred at room temperature for 3 h. The reaction mixture was quenched with sat. $\mathrm{NaHCO}_{3}$ solution $(10 \mathrm{~mL})$, filtered and washed with $\mathrm{CH}_{2} \mathrm{Cl}_{2}(30$ $\mathrm{mL})$. The filtrate was washed with water $(30 \mathrm{~mL})$, brine $(30 \mathrm{~mL})$, dried $\left(\mathrm{Na}_{2} \mathrm{SO}_{4}\right)$ and evaporated under reduced pressure. The residue was purified by column chromatography (60-120 Silica gel, 20\% EtOAc in pet. ether) to furnish (10) (1.78 g, 86\%). [ $\alpha]_{\mathrm{D}}^{25}+46.1$ (c 0.9, $\mathrm{CHCl}_{3}$ ); IR (neat): 3470, 2983, 2927, 1612, 1513, 1458, 1374, 1248, 1173, 1090, $1042 \mathrm{~cm}^{-1} ;{ }^{1} \mathrm{H}$ NMR $\left(300 \mathrm{MHz}, \mathrm{CDCl}_{3}\right): \delta$ 7.37-7.22 (m, 5H), $4.59(\mathrm{~s}, 2 \mathrm{H}), 3.66-3.54(\mathrm{~m}, 1 \mathrm{H}), 3.48(\mathrm{t}$, $2 \mathrm{H}$, J $6.5 \mathrm{~Hz}), 3.33(\mathrm{~m}, 1 \mathrm{H}), 2.98$ (brs, $1 \mathrm{H}), 1.68-1.49(\mathrm{~m}, 5 \mathrm{H}), 1.47-1.30(\mathrm{~m}, 2 \mathrm{H}), 1.22(\mathrm{~d}, 3 \mathrm{H}, J 6.3 \mathrm{~Hz}), 1.17-1.09$ $(\mathrm{m}, 1 \mathrm{H}), 0.83(\mathrm{~s}, 9 \mathrm{H}), 0.12(\mathrm{~s}, 3 \mathrm{H}), 0.01(\mathrm{~s}, 3 \mathrm{H}) .{ }^{13} \mathrm{C} \mathrm{NMR}\left(\mathrm{CDCl}_{3}, 75 \mathrm{MHz}\right): 139.3,129.2,128.8,128.3,79.2,72.4$, $67.6,61.9,39.2,33.4,33.3,31.2,26.8,23.9,19.3,-4.2,-3.9$; ESIMS: $389(\mathrm{M}+\mathrm{Na})^{+}$. HRMS (ESI): $\mathrm{m} / \mathrm{z}$ calcd for $\mathrm{C}_{21} \mathrm{H}_{38} \mathrm{O}_{3} \mathrm{SiNa}$ : 389.2486; found: $389.2488[\mathrm{M}+\mathrm{Na}]^{+}$.

(4S,7S)-4-(Benzyloxy)-7-(tert-butyldimethylsilyloxy)octanoic acid (11). To a stirred solution of the octanol (10) $(1.55 \mathrm{~g}, 4.23 \mathrm{mmol})$ in $\mathrm{CH}_{2} \mathrm{Cl}_{2}: \mathrm{H}_{2} \mathrm{O}(1: 1,10 \mathrm{~mL})$, TEMPO $(0.19 \mathrm{~g}, 1.27 \mathrm{mmol})$ and [bis(acetoxy)iodo]benzene $(0.40 \mathrm{~g}, 1.27 \mathrm{mmol})$ were added at $0{ }^{\circ} \mathrm{C}$ and stirred for $1 \mathrm{~h}$. The reaction mixture was diluted with water (20 $\mathrm{mL})$ and extracted with $\mathrm{CH}_{2} \mathrm{Cl}_{2}(2 \times 30 \mathrm{~mL})$. The combined organic layers were washed with brine $(20 \mathrm{~mL})$, dried $\left(\mathrm{Na}_{2} \mathrm{SO}_{4}\right)$, evaporated and the residue purified by column chromatography (silica gel, 60-120 mesh, 30\% EtOAc in pet. ether) to give acid (11) $(1.2 \mathrm{~g}, 75 \%)$ as a colorless gummy oil. $[\alpha]_{\mathrm{D}}^{25}=-105.3\left(\mathrm{c} 0.25, \mathrm{CHCl}_{3}\right) ; \mathrm{IR}$ 
(neat): $3435,2958,2855,1727,1614,1520,1369,1299,1174,1012 \mathrm{~cm}^{-1} ;{ }^{1} \mathrm{H} \mathrm{NMR}\left(300 \mathrm{MHz}, \mathrm{CDCl}_{3}\right): \delta 7.41-$ $7.36(\mathrm{~m}, 5 \mathrm{H}), 4.54(\mathrm{~d}, 1 \mathrm{H}, J 10.8 \mathrm{~Hz}), 4.46(\mathrm{~d}, 1 \mathrm{H}, J 10.8 \mathrm{~Hz}), 3.61-3.50(\mathrm{~m}, 1 \mathrm{H}), 3.42-3.37(\mathrm{~m}, 1 \mathrm{H}), 2.36(\mathrm{t}, J 7.1$ $\mathrm{Hz}, 2 \mathrm{H}), 1.59-1.33(\mathrm{~m}, 6 \mathrm{H}), 1.21(\mathrm{~d}, 3 \mathrm{H}, \mathrm{J} 6.8 \mathrm{~Hz}), 0.91(\mathrm{~s}, 9 \mathrm{H}), 0.13(\mathrm{~s}, 3 \mathrm{H}), 0.06(\mathrm{~s}, 3 \mathrm{H}) .{ }^{13} \mathrm{C} \mathrm{NMR}(\mathrm{CDCl}, 75$ $\mathrm{MHz}$ : $177.2,139.8,129.6,129.0,128.7,79.3,72.7,67.6,38.3,33.7,30.3,29.6,26.9,24.4,19.7,-4.3,-3.9$. ESIMS: $403(\mathrm{M}+\mathrm{Na})^{+}$. HRMS (ESI): $\mathrm{m} / \mathrm{z}$ calcd for $\mathrm{C}_{21} \mathrm{H}_{36} \mathrm{O}_{4} \mathrm{SiNa}$ : 403.2283; found: 403.2286 [M+Na] ${ }^{+}$.

$(4 S, 7 S)$-4-(Benzyloxy)-7-hydroxyoctanoic acid (2). To a cooled $\left(0^{\circ} \mathrm{C}\right)$ solution of the octanoic acid (11) (1.1 g, $2.89 \mathrm{mmol})$ in dry THF (15 $\mathrm{mL})$ under nitrogen atmosphere, TBAF $(4.3 \mathrm{~mL}, 4.34 \mathrm{mmol})$ was added and stirred for $3 \mathrm{~h}$. After completion of the reaction, the reaction mixture was diluted with water $(5 \mathrm{~mL})$ and extracted with ethyl acetate $(2 \times 50 \mathrm{~mL})$. The combined organic layers were washed with water $(2 \times 10 \mathrm{~mL})$, brine $(10$ $\mathrm{mL})$, dried $\left(\mathrm{Na}_{2} \mathrm{SO}_{4}\right)$, evaporated, and the residue was purified by column chromatography (60-120 silica gel, $55 \%$ EtOAc in pet. ether) to give (2) $(0.66 \mathrm{~g}, 86 \%)$ as a white solid which was used for the next step without purification. $[\alpha]_{\mathrm{D}}^{25}=-16.8$ (c $0.25, \mathrm{CHCl}_{3}$ ); IR (neat): 3490, 2976, 2840,1725, 1619, 1520, 1360, 1268, 1175, 1012 $\mathrm{cm}^{-1} ;{ }^{1} \mathrm{H}$ NMR $\left(300 \mathrm{MHz}, \mathrm{CDCl}_{3}\right): \delta$ 7.36-7.26 (m, 5H), $4.47(\mathrm{~s}, 2 \mathrm{H}), 3.77-3.68(\mathrm{~m}, 1 \mathrm{H}), 3.48(\mathrm{~m}, 1 \mathrm{H}), 3.04$ (brs, $1 \mathrm{H}), 2.34(\mathrm{t}, J 6.6 \mathrm{~Hz}, 2 \mathrm{H}), 1.71-1.64(\mathrm{~m}, 1 \mathrm{H}), 1.57-1.38(\mathrm{~m}, 5 \mathrm{H}), 1.19(\mathrm{~d}, 3 \mathrm{H}, J 6.6 \mathrm{~Hz}) .{ }^{13} \mathrm{C} \mathrm{NMR}(\mathrm{CDCl}, 75 \mathrm{MHz})$ : 176.6, 139.3, 129.3, 129.0, 128.6, 80.1, 72.4, 66.6, 36.3, 32.1, 30.4, 29.3, 23.8. ESIMS: 289 (M+ Na) ${ }^{+}$. HRMS (ESI): $\mathrm{m} / \mathrm{z}$ calcd for $\mathrm{C}_{15} \mathrm{H}_{22} \mathrm{O}_{4} \mathrm{Na}$ : 289.1416; found: $289.1421[\mathrm{M}+\mathrm{Na}]^{+}$.

(5S,8R,13S,16R)-5,13-Bis(benzyloxy)-8,16-dimethyl-1,9-dioxacyclohexadecane-2,10-dione (12). To a solution of the hydroxy acid (2) $(0.26 \mathrm{~g}, 0.97 \mathrm{mmol})$ and $\mathrm{Ph}_{3} \mathrm{P}(1.28 \mathrm{~g}, 4.88 \mathrm{mmol})$ in toluene:THF $(10: 1,260 \mathrm{~mL}), \mathrm{DEAD}$ $(2.76 \mathrm{~mL}, 17.46 \mathrm{mmol})$ was added at $-20{ }^{\circ} \mathrm{C}$ and stirred under $\mathrm{N}_{2}$ atmosphere for $10 \mathrm{~h}$. Solvent was evaporated under reduced pressure, and the residue purified by column chromatography (60-120 silica gel, 15\% EtOAc in pet. ether) to afford (12) $(0.14 \mathrm{~g}, 59 \%)$ as a colorless oil. $[\alpha]_{\mathrm{D}}^{25}+7.9\left(c 1.03, \mathrm{CHCl}_{3}\right) ;{ }^{1} \mathrm{H} \mathrm{NMR}\left(\mathrm{CDCl}_{3}, 300 \mathrm{MHz}\right): \delta$ 7.35-7.22 (m, 10H), 5.03-4.91 (m, 2H), $4.51(\mathrm{~s}, 4 \mathrm{H}), 3.58-3.41(\mathrm{~m}, 2 \mathrm{H}), 2.34(\mathrm{t}, 4 \mathrm{H}, J 6.6 \mathrm{~Hz}), 1.79-1.60(\mathrm{~m}, 8 \mathrm{H})$, 1.57-1.41 (m, 2H), 1.37-1.29 (m, 2H), $1.19(\mathrm{~d}, J 6.1 \mathrm{~Hz}, 6 \mathrm{H}) ;{ }^{13} \mathrm{C} \mathrm{NMR}\left(\mathrm{CDCl}_{3}, 75 \mathrm{MHz}\right): 177.9,139.4,129.1$, 128.7, 128.3, 128.0, 79.4, 73.1, 69.0, 33.2, 32.4, 29.8, 29.4, 23.2; ESIMS: 519 (M+ Na) ${ }^{+}$. HRMS (ESI): $\mathrm{m} / \mathrm{z}$ calcd for $\mathrm{C}_{30} \mathrm{H}_{40} \mathrm{O}_{6} \mathrm{Na}: 519.2744$; found: $519.2751[\mathrm{M}+\mathrm{Na}]^{+}$.

(-)-1-Tetrahydropyrenophorol (1). To a stirred solution of the dilactone (12) $(0.090 \mathrm{~g}, 0.18 \mathrm{mmol})$ in dichloromethane $(2 \mathrm{~mL}), \mathrm{TiCl}_{4}(0.04 \mathrm{~mL}, 0.36 \mathrm{mmol})$ in dichloromethane was added at $0{ }^{\circ} \mathrm{C}$ and stirred for $1 \mathrm{~h}$. Sat. aq. $\mathrm{NaHCO}_{3}$ solution $(10 \mathrm{~mL})$ was added and the mixture extracted with dichloromethane $(3 \times 10 \mathrm{~mL})$. The combined organic layers were washed with water $(15 \mathrm{~mL})$, brine $(10 \mathrm{~mL})$, dried $\left(\mathrm{Na}_{2} \mathrm{SO}_{4}\right)$ and concentrated. The crude residue was purified by column chromatography (silica gel, 60-120 mesh, 30\% EtOAc in pet. ether) to afford the tetrahydropyrenophorol (1) (44 mg) in 77\% yield as a white solid. M.p. $126-128{ }^{\circ} \mathrm{C}$; $[\alpha]_{\mathrm{D}}^{25}-70.3$ (c 0.54, $\left.\mathrm{CHCl}_{3}\right) ;{ }^{1} \mathrm{H}$ NMR $\left(300 \mathrm{MHz}, \mathrm{CDCl}_{3}\right): \delta$ 5.05-4.99 (m, 2H), 3.59-3.51 (m, $\left.2 \mathrm{H}\right), 2.47-2.34(\mathrm{~m}, 4 \mathrm{H}), 1.91-1.78$ $(\mathrm{m}, 4 \mathrm{H}), 1.75-1.63(\mathrm{~m}, 4 \mathrm{H}), 1.52-1.44(\mathrm{~m}, 2 \mathrm{H}), 1.38-1.33(\mathrm{~m}, 2 \mathrm{H}), 1.22(\mathrm{~d}, J 6.1 \mathrm{~Hz}, 6 \mathrm{H}) ;{ }^{13} \mathrm{C} \mathrm{NMR}(75 \mathrm{MHz}$, $\left.\mathrm{CDCl}_{3}\right): \delta 173.4,69.8,68.1,32.9,31.0,30.8,30.6,20.1$; ESIMS: $317(\mathrm{M}+\mathrm{H})^{+}$. HRMS (ESI): $\mathrm{m} / \mathrm{z}$ calcd for $\mathrm{C}_{16} \mathrm{H}_{28} \mathrm{O}_{6} \mathrm{Na}: 339.1785$; found: $339.1788[\mathrm{M}+\mathrm{Na}]^{+}$.

\section{Acknowledgements}

The authors are thankful to GVK Bio sciences and CSIR, New Delhi for constant encouragement in providing laboratory facilities and analytical data. 


\section{Supplementary Material}

Copies of ${ }^{1} \mathrm{H}$ and ${ }^{13} \mathrm{C}$ NMR spectra associated with this paper can be found in the online version.

\section{References}

1. Kis, Z.; Furger, P.; Sigg, H. Experientia 1969, 25, 123. https://doi.org/10.1007/BF01899073

2. Krohn, K.; Farooq, U.; Flörke, U.; Schulz, B.; Draeger, S.; Pescitelli, P.; Salvadori, G. ; Antus, S.; Kurtán, T. Eur. J. Org. Chem. 2007, 3206. https://doi.org/10.1002/ejoc.200601128

3. Nozoe,S.; Hira, K.; Tsuda, K.; ishibashi, K.; Grove, J.F. Tetrahedron Lett. 1965, 4675.

4. Kind, R.; Zeeck, A.; Grabley, S.; Thiericke, R.; Zerlin, M. J. Nat. Prod. 1996, 59, 539. https://doi.org/10.1002/ejoc.200601128

5. Ghisalberti, E. L.; Hargreaves, J. R.; Skelton, B. W.; White, A. H.; Aust. J. Chem. 2002, 55, 233. https://doi.org/10.1071/CH01197

6. Christner, C.; Kullertz, G.; Fischer, G.; Zerlin, M.; Grabley, S.; Thiericke, R.; Taddei, A.; Zeeck, A. J. Antibiot. 1998, 51, 368.

https://doi.org/10.7164/antibiotics.51.368

7. Kastanias, M. A.; Chrysayi-Tokousbalides, M. Pest Manage. Sci. 2000, 56, 227. https://doi.org/10.1002/(SICI)1526-4998(200003)56:3<227::AID-PS115>3.0.CO;2-A

8. Kastanias, M. A.; Chrysayi-Tokousbalides, M. J. Agric. Food Chem. 2005, 53, 5943. https://doi.org/10.1021/if050792m

9. Sugawara, F.; Strobel, G. A. Plant Sci. 1986, 43, 1. https://doi.org/10.1016/0168-9452(86)90099-3

10. Pratapareddy, B.; Sreenivasulu, R.; Thota, P.; Hatti, I.; Basaveswara Rao, M. V.; Naresh Kumar, V.; Ramesh Raju, R. Monatsh. Chem. ,2017 148, 751. https://doi.org/10.1007/s00706-016-1754-2

11. Trost, B. M.; Quintard, A. Angew. Chem. Int. Ed. 2012, 51, 6704. https://doi.org/10.1002/anie.201203035

12. Madala, M.; Raman, B.; Sastry, K.V.; Sridhar, M. Monatsh. Chem. 2016, 147, 1985. https://doi.org/10.1007/s00706-016-1682-1

13. Paul Raj, I. V.; Sudalai, A. Tetrahedron Lett. 2008, 49, 2646. https://doi.org/10.1016/j.tetlet.2008.02.064

14. Mori, k.; Sakai, T. Liebigs Annalen der Chemie 1988, 25, 13.

15. Gerlach, H.; Gertle, K.; Thalmann, A. Helv. Chim. Acta 1977, 60, 2860. https://doi.org/10.1016/i.tetlet.2008.02.064 